\title{
Focused acute medicine ultrasound (FAMUS): curriculum outline of a new ultrasound standard for acute internal medicine
}

\author{
Authors: Nicholas Smallwood, ${ }^{1}$ Martin Dachsel ${ }^{2}$ and Alastair Gilmore ${ }^{3}$
}

\begin{abstract}
Aims
Point-of-care ultrasound (POCU) is becoming an increasingly popular aide to traditional examination techniques at the bedside. A number of specialties have developed their own curricula (FAST in emergency medicine, CUSIC in intensive care medicine, for example). A working group was set up to develop a POCU standard for acute internal medicine and we present here an outline of the FAMUS curriculum. This standard can be undertaken by anyone with an interest in POCU, and is relevant to all clinicians with a commitment to the care of the acutely unwell adult patient. It is endorsed by the Society for Acute Medicine (SAM), and accredited as a specialist skill in ultrasound for acute internal medicine (AIM) trainees.
\end{abstract}

\section{Methods}

A working group comprised clinicians from radiology, acute and intensive care medicine with experience in POCU. A survey of attitudes to POCU on the acute medical unit was undertaken, and subsequently through a series of face-to-face and teleconference meetings, a POCU standard and training pathway was devised.

\section{Results}

The FAMUS curriculum comprises a theoretical component and three modular practical components (thoracic, abdominal/ renal and DVT/vascular). These modular components reflect the areas of interest identified in a nationwide survey of attitudes and experience in POCU. Each practical component involves directly supervised and then mentored practice, and once the trainee is competent, an assessment of completion of training in that area must be completed. Candidates must accomplish all four components before their training is complete and they become FAMUS accredited.

Authors: ${ }^{1}$ Worthing Hospital, Worthing, UK; ${ }^{2}$ East Surrey Hospital, Redhill, UK; ${ }^{3}$ Arrowe Park Hospital, Wirral, UK

\section{Conclusions}

We present here a new POCU training curriculum for clinicians looking after the acutely unwell adult medical patient. It is the first UK training standard developed specifically for bedside assessment of the general medicine patient population. Although developed in response to a need within AIM, it is expected to appeal to a wide range of clinicians who are involved in the care of the acutely unwell patient (for example, anyone with a significant general internal medicine commitment). It provides skills in the assessment of patients with respiratory failure, acute kidney injury, those with abdominal free fluid and in suspected DVT. In addition, it will provide the skills for peripheral vascular cannulation under ultrasound guidance.

FAMUS accreditation has been approved by the AIM training committee as a specialist skill in ultrasound, and it is hoped in time will become a core part of the AIM curriculum.

\section{Conflict of interest statement}

There are no conflicts of interest to declare. 